DOI: 10.12731/2658-6649-2019-11-4-56-73

УДК 572.512:616.24-036.12:616.12-008.331.1

\title{
АНТРОПОМЕТРИЧЕСКИЕ ОСОБЕННОСТИ И КОМПОНЕНТНЫЙ СОСТАВ МАССЫ ТЕЛА У МУЖЧИН С ХРОНИЧЕСКОЙ ОБСТРУКТИВНОЙ БОЛЕЗНЬЮ ЛЕГКИХ В УСЛОВИЯХ КОМОРБИДНОСТИ
}

\author{
Козлов Е.В., Яскевич Р.А., Москаленко О.Л., Кочергина К.Н.
}

Цель: Изучение и сравнительный анализ компонентного состава массы тела и антропометрических показателей у мужчин с хронической болезнью легких (ХОБЛ) при коморбидности с артериальной гипертонией (АГ).

Материалы и методы. В исследование было включено 136 пациентов мужского пола, средний возраст 61 лет. Из них пациентов с ХОБЛ в сочетании с АГ- 63 человека, пациентов с ХОБЛ без АГ 29 человек. Группу сравнения составили 44 паџиента с АГ без ХОБЛ. Протокол исследования включал: клинические, инструментальные и лабораторные методы исследования.

Результаты. В ходе проведенного исследования были выделены закономерности распределения конституцииональных типов у мужчин с ХОБЛ в условиях коморбидности, а также установлень особенности антропометрических показателей и компонентного состава массы тела в исследуемых группах. Особенностью распределения мужчин по конституичональным типам является преобладание лиц с грудным конституциональным типом среди мужчин с ХОБЛ и преобладание лиц с мускульным конституциональным типом среди мужчин с АГ и ХОБЛ и АГ. Получень данные о росто-весовых параметрах, компонентном составе тела у представителей с ХОБЛ в зависимости от степени тяжести заболевания. При прогрессировании ХОБЛ отмечается тенденция к уменьшению ИМТ и снижению жировой и мышечной компонент массы тела.

Заключение. Установлено, что в при прогрессирования ХОБЛ уменьшаются значения ИМТ, с параллельным увеличением доли паџиентов с нормальной массой тела, за счет уменьшения доли больных с избыточной массой тела и ожирением. 
Ключевые слова: хроническая обструктивная болезнь легких; артериальная гипертония; коморбидность; соматотип; масса тела.

\title{
ANTHROPOMETRIC FEATURES AND COMPONENT STRUCTURE OF BODY MASS IN MEN WITH CHRONIC OBSTRUCTIVE PULMONARY DISEASE IN CONDITIONS OF COMORBIDITY
}

\author{
Kozlov E.V., Yaskevich R.A., Moskalenko O.L., Kochergina K.N.
}

The purpose of the study. The study and comparative analysis of the component composition of body weight and anthropometric parameters in men with chronic lung disease (COPD) with comorbidity with arterial hypertension (AH).

Materials and methods. The study included 136 male patients, mean age 61 years. Of these, patients with COPD in combination with hypertension - 63 people, patients with COPD without hypertension 29 people. The comparison group consisted of 44 patients with hypertension without COPD. The study protocol included: clinical, instrumental and laboratory research methods.

Results. In the course of the study, the patterns of distribution of constitutional types among men with COPD under conditions of comorbidity were identified, and the characteristics of anthropometric indices and component composition of body weight in the studied groups were established. A distinctive feature of the distribution of men by constitutional types is the predominance of persons with breast constitutional type among men with COPD and the predominance of persons with muscular constitutional type among men with hypertension and COPD and hypertension. Data were obtained on height-weight parameters, component composition of the body in representatives with COPD, depending on the severity of the disease. With the progression of COPD, there is a tendency to a decrease in BMI and a decrease in the fat and muscle component of body weight.

Conclusion. It has been established that during the progression of COPD, the values of BMI decrease, with a parallel increase in the proportion of patients with normal body weight, due to a decrease in the proportion of patients with overweight and obesity.

Keywords: chronic obstructive pulmonary disease; arterial hypertension; comorbidity; somatotype; body weight. 


\section{Введение}

По оценкам Всемирной Организации Здравоохранения (ВО3), хрони-

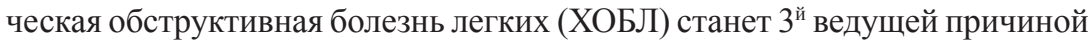
смерти в мире к 2030 году [5, с. 23-25; 10, с. 180-199]. В настоящее время ХОБЛ рассматривается как системное заболевание, часто ассоциирующееся с сердечно-сосудистыми заболеваниями, метаболическим синдромом (МC) и сахарным диабетом (СД) [3, с. 127-134; 10, с. 180-199; 16, с. 109118]. Довольно часто встречается коморбидность артериальной гипертонии (АГ) и ХОБЛ [10, с. 180-199; 16, с. 109-118; 18, с. 5-34].

Индивидуально-типологические особенности человека могут служить маркерами, отражающие особенности клинических проявлений и позволяющие прогнозировать тяжесть течения тех или иных заболеваний [13, c. $10-14 ; 15$, с. $14-19 ; 19$, с. 125]. Показано, что конституциональная диагностика выявляет коррелятивные связи между антропометрическими особенностями человека и выраженностью клинических проявлений той или иной патологии $[1$, с. $453-459 ; 6$, с. 19-21; 7, с. 21-23; 8, с. 252-256; 29, c. 47-63]. Обнаружено неблагоприятное влияние снижения индекса массы тела (ИМТ) у пациентов, страдающих ХОБЛ, на течение заболевания, частоту обострений, толерантность к физической нагрузке [11, с. 14-19; 12, c. $42-48 ; 14$, с. $60-63 ; 24$, с. 533-538; 27, с. 424-30]. Избыток массы тела, ожирение также нередко наблюдаются у пациентов с ХОБЛ, что приводит не только к снижению показателей, характеризующих функцию внешнего дыхания, но и к развитию сердечно-сосудистой патологии и нарушению углеводного обмена $[1$, с. $453-459 ; 16$, с. $109-118 ; 17$, с. 439-447; 28 , c. 15-22]. Для объективной оценки соматического типа в комплекс соматодиагностики обязательно должен быть включен анализ компонентного состава тела [21, с. 43-52]. Соотношение мышечного, костного и жирового компонентов в значительной степени зависит от среды обитания, особенностей питания, физической активности и воздействия стрессовых факторов $[4$, с. $330-333 ; 29$, с. $47-63 ; 22$, с. 10-34]. Значимость изучения соотношения указанных компонентов в теории и практике конституционологии отмечалась рядом авторов $[4$, с. 330-333; 11 , с. 14-19; 26, с. е0180928; 27, с. 424-30]. Снижение массы тела и, в частности, мышечной массы - независимые предикторы смертности при ХОБЛ. Вместе с тем, нередки случаи снижения мышечной массы при нормальном или повышенном ИМТ [26, с. е0180928]. Снижение безжировой массы тела (главным образом, за счет мышечной ткани) - значимая проблема у $35 \%$ пациентов с тяжелой и $20 \%$ со средней степенью ХОБЛ [14, с. 60-63]. Известно, что 
индекс массы тела (ИМТ) является наиболее распространенным методом выявления избыточной массы тела и ожирения. Данный показатель не разграничивает массу жировой и мышечной ткани, однако в высокой степени ассоциирован с ними [9, с. 26-46; 12 , с. 42-48; 27, с. 424-30; 29, с. 47-63]. Влияние средовых факторов наиболее динамично отражают изменения жирового компонента массы тела. Аномалии содержания и особенности распределения жира в организме человека являются диагностически важным признаком многих патологических состояний [26, с. е0180928].

В связи с этим большой интерес представляет изучение антропометрических особенностей и компонентного состава массы тела у пациентов с ХОБЛ для комплексной оценки состояния и персонификации лечения, а нормализация распределения и количества жировой ткани значимо снизит риски сопутствующих заболеваний и в итоге приведет к увеличению продолжительность жизни пациентов [11, с. 14-19].

\section{Цель исследования}

Изучение и сравнительный анализ компонентного состава массы тела и антропометрических показателей у мужчин с ХОБЛ при коморбидности с АГ.

\section{Материалы и методы}

В исследование было включено 136 пациентов мужского пола, средний возраст 61 [57-68] лет. Из них пациенты с ХОБЛ в сочетании с АГ - 63 чел. (группа №2) средний возраст 64 [58-70] лет, пациентов с ХОБЛ без АГ 29 чел. (группа №1) средний возраст 59 [52-69] лет. Группу сравнения составили 44 пациента с АГ без ХОБЛ (группа №3), средний возраст 60 [58-64] лет.

Протокол исследования включал: клинические, инструментальные и лабораторные методы исследования. Верификация диагноза ХОБЛ и оценка степени тяжести проводилась в соответствии с критериями GOLD (Global Initiative for Chronic Obstructive Lung Disease, update 2014) [5, c. 23-25]. Верификацию АГ проводили согласно Рекомендациям по диагностике и лечению АГ Всероссийского научного общества кардиологов (BНОК,2013) [18, c. 5-34].

Антропометрическое исследование проводилось по общепринятым правилам антропометрических измерений и включало в себя определение продольных, поперечных, обхватных размеров тела, его массы и силовые показатели. По методике J. Matiegka (1921) определяли толщину кожно-жировых складок, дистальные костные диаметры (плеча, предплечья, бедра и голени), а также обхваты плеча, предплечья, бедра и голени. 
Для определения компонентного состава массы тела использовали методику фракционирования массы тела на мышечный, костный и жировой компоненты. Вычисление жирового, мышечного и костного компонентов массы тела проводили по специальным формулам. Соматотипирование проводилось по методу Бунака В.В. (1931) [2, с. 2-7] в модификации Чтецова В.П. с соавт. (1978) [20, с. 3-22], основывалось на 5-бальной оценке трех компонентов состава тела человека: жирового, мышечного и костного. При определении соматотипа мужчин использовали терминологию, предложенную Бунаком В.В. (1931): грудной, грудно-мускульный, мускульно-грудной, мускульный, мускульно-брюшной, брюшно-мускульный, брюшной, грудно-брюшной, брюшно-грудной типы и т.п. [2, с. 2-7]. Соматотипы определялись согласно схеме возможного сочетания баллов.

Индекс массы тела (ИМТ) рассчитывался по формуле: вес (кг)/рост $\left(\mathrm{m}^{2}\right)$. За ожирение принимали ИМТ $\geq 30$ кг/ $\mathrm{M}^{2}$. Обхват талии (ОТ) измеряли по стандартной методике. Абдоминальное ожирение для мужчин определяли при ОТ $\geq 102$ см. Индекс висцерального ожирения (ИВО) для мужчин рассчитывали по формуле: ИВО $=[$ ОТ/39,68+(1,88•ИМТ $)] \cdot($ ТГ 1,03$) \cdot$ (1,31/ЛВП) [23, с. 920-922]. Индекс продукта накопления липидов (ИПНЛ) для мужчин рассчитывали по формуле: ИПНЛ $=(\mathrm{OT-65}) \cdot$ ТГ [25, с. 26].

Обработку полученных данных проводили при помощи пакета прикладных программ Statistica 6.0 (Statsoft Inc., США). Количественные значения представлялись в виде медианы $(\mathrm{Me})$ и межквартильного интервала $\left(\mathrm{Q}_{25}-\mathrm{Q}_{75}\right)$. Достоверность различий между показателями независимых выборок оценивали по непараметрическому критерию Манна-Уитни. При сравнении групп по качественному признаку использовали критерий $\chi^{2}$. Различия считали статистически значимыми при $\mathrm{p}<0,05$.

\section{Результаты и обсуждение}

Для определения индивидуально-типологических особенностей обследованные мужчины были разделены на основании антропометрических данных по соматотипам (табл.1). Грудной тип конституции среди мужчин с ХОБЛ встречался в 11 случаях из 29 (37,9\%), 7 мужчин имели брюшной тип конституции (24,2\%), мускульной тип - у 1 человека (3,5\%). В 10 случаях $(34,5 \%)$ принадлежность к остальным конституциональным группам установить не удалось (неопределенная конституция). Обладателей мускульного типа было достоверно меньше в сравнении с мужчинами, имеющими грудной тип $\left(\chi^{2}=10,5, \mathrm{df}=1, \mathrm{p}=0,001\right)$, брюшной тип $\left(\chi^{2}=5,22, \mathrm{df}=1\right.$, $\mathrm{p}=0,022)$ и неопределенный тип $\left(\chi^{2}=9,09, \mathrm{df}=1, \mathrm{p}=0,003\right)$. 
Таблица 1.

Частота встречаемости различных конституциональных типов среди обследованных мужчин $\left(\mathrm{Me}\left[\mathrm{Q}_{25}-\mathrm{Q}_{75}\right]\right)$

\begin{tabular}{|c|c|c|c|c|}
\hline \multirow[t]{2}{*}{ Соматотип } & $\begin{array}{c}\text { a) ХОБЛ }(+), \text { АГ } \\
(-)(n=29)\end{array}$ & $\begin{array}{c}\text { b) ХОБЛ+АГ } \\
(\mathrm{n}=63)\end{array}$ & $\begin{array}{c}\text { c) АГ (+), ХОБЛ } \\
(-)(n=44)\end{array}$ & \multirow[t]{2}{*}{$\mathrm{p}$} \\
\hline & Абс.(\%) & Абс.(\%) & Абс.(\%) & \\
\hline 1. Грудной & $11(37,9)$ & $16(25,4)$ & $7(15,9)$ & $\begin{array}{l}\mathrm{p}_{\mathrm{a}, \mathrm{b}}=0,220 \\
\mathbf{p}_{\mathrm{a}, \mathrm{c}}=\mathbf{0 , 0 3 3} \\
\mathrm{p}_{\mathrm{b}, \mathrm{c}}=0,239\end{array}$ \\
\hline $\begin{array}{l}\text { 2. Му- } \\
\text { скульный }\end{array}$ & $1(3,5)$ & $10(15,9)$ & $18(40,9)$ & $\begin{array}{l}\mathbf{p}_{\mathrm{a}, \mathrm{b}}=0,088 \\
\mathbf{p}_{\mathrm{a}, \mathrm{c}}=\mathbf{0 , 0 0 1} \\
\mathbf{p}_{\mathrm{b}, \mathrm{c}}=\mathbf{0 , 0 4 5}\end{array}$ \\
\hline $\begin{array}{l}\text { 3. Брюш- } \\
\text { ной }\end{array}$ & $7(24,2)$ & $22(34,9)$ & $15(34,1)$ & $\begin{array}{l}\mathrm{p}_{\mathrm{a}, \mathrm{b}}=0,301 \\
\mathrm{p}_{\mathrm{a}, \mathrm{c}}=0,365 \\
\mathrm{p}_{\mathrm{b}, \mathrm{c}}=0,929\end{array}$ \\
\hline $\begin{array}{l}\text { 4. Неопре-- } \\
\text { деленный }\end{array}$ & $10(34,5)$ & $15(23,8)$ & $4(9,1)$ & $\begin{array}{l}p_{a, b}=0,285 \\
p_{a, \mathbf{c}}=\mathbf{0 , 0 0 7} \\
\mathbf{p}_{b, \mathbf{c}}=\mathbf{0 , 0 5 0}\end{array}$ \\
\hline $\mathrm{p}$ & $\begin{array}{ll}\mathbf{p}_{1,2}=\mathbf{0 , 0 0 1} & \mathbf{P}_{2,3}=\mathbf{0 , 0 2 2} \\
\mathbf{p}_{1,3}=0,256 & \mathbf{p}_{2,4}=\mathbf{0 , 0 0 3} \\
\mathbf{p}_{1,4}=0,785 & \mathrm{p}_{3,4}=0,387\end{array}$ & $\begin{array}{ll}\mathrm{p}_{1,2}=0,187 & \mathbf{P}_{2,3}=\mathbf{0 , 0 1 4} \\
\mathrm{p}_{1,3}=0,244 & \mathrm{p}_{2,4}=0,264 \\
\mathrm{p}_{1,4}=0,836 & \mathrm{p}_{3,4}=0,171 \\
\end{array}$ & $\begin{array}{ll}\mathbf{p}_{1,2}=\mathbf{0 , 0 0 9} & \mathrm{P}_{2,3}=0,509 \\
\mathbf{p}_{1,3}=\mathbf{0 , 0 4 9} & \mathbf{p}_{2,4}=\mathbf{0 , 0 0 1} \\
\mathrm{p}_{1,4}=0,334 & \mathbf{p}_{3,4}=\mathbf{0 , 0 0 4}\end{array}$ & \\
\hline
\end{tabular}

Среди мужчин с ХОБЛ и АГ большинство мужчин имели брюшной тип конституции - 22 из 63 случаев (34,9\%), грудной тип конституции встречался у 16 мужчин (25,4\%), мускульной тип - у 10 человек (15,9\%). В 15 случаях $(23,8 \%)$ отмечалась неопределенная конституция. Статистически значимые различия наблюдались только между пациентами с мускульным и брюшным типом конституции $\left(\chi^{2}=6,03, \mathrm{df}=1, \mathrm{p}=0,014\right)$.

В группе мужчин, страдающих ХОБЛ без артериальной гипертонии, значительно чаще встречались представители мускульного типа - 18 человек $(40,9 \%)$ и брюшного типа конституции - 15 мужчин $(34,1 \%)$. Грудной тип имели 7 мужчин из 44 (15,9\%), что достоверно меньше чем обладателей мускульного $\left(\chi^{2}=6,76, \mathrm{df}=1, \mathrm{p}=0,009\right)$ и брюшного типов $\left(\chi^{2}=3,88, \mathrm{df}=1, \mathrm{p}=0,049\right)$.

Резюмируя вышеизложенное, следует отметить, что грудной тип чаще встречался среди мужчин с ХОБЛ в отличие от больных с АГ $\left(\chi^{2}=4,56\right.$, $\mathrm{df}=1, \mathrm{p}=0,033)$, а мускульный тип, напротив, преобладал среди мужчин, страдающих АГ $\left(\chi^{2}=12,7, \mathrm{df}=1, \mathrm{p}=0,001\right)$ и ХОБЛ и АГ $\left(\chi^{2}=4,01, \mathrm{df}=1\right.$, $\mathrm{p}=0,045)$, в сравнении с группой ХОБЛ. Среди мужчин с АГ и ХОБЛ и АГ несколько чаще, на уровне тенденции, встречались представители брюшного типа конституции. Полученные результаты согласуются с данными 
Деревянных Е.В. с соавт. $(2016,2018)$ [6, с. 19-21; 7, с. 21-23; 8, с. 252-256] согласно которым высокая частота встречаемости АГ среди мужчин медицинских работников г. Красноярска отмечалась у лиц с брюшным соматотипом - 52,2\%, а наименьшая с неопределенным - 11,5\% [8, с. 252-256].

Проведен анализ уровней артериального давления (АД), компонентов массы тела и антропометрических индексов у мужчин с ХОБЛ и АГ (табл. 2). Средние показатели САД и ДАД имели статистически значимые различия в группах мужчин с ХОБЛ и ХОБЛ+АГ $(\mathrm{U}=219,5, \mathrm{Z}=5,83$, $\mathrm{p}=0,001)$ и с ХОБЛ и $\mathrm{AГ}(\mathrm{U}=262,0, \mathrm{Z}=-4,23, \mathrm{p}=0,001)$. У лиц с АГ отмечались наибольшие значения массы тела (82,5 кг) и ИМТ $(27,96$ кг/м²) в срав-

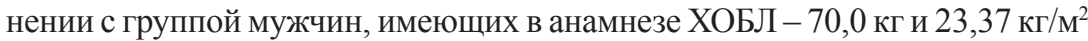
$(\mathrm{U}=307,5, \mathrm{Z}=-3,72, \mathrm{p}=0,001)$. Наименьшие значения показателей жировой, мышечной и костной ткани отмечались у больных с ХОБЛ: количество жировой ткани $-10,56$ кг, мышечной ткани $-27,05$ кг, костной ткани $-11,03$ кг. Наблюдается достоверное различие этих показателей с аналогичными в группе мужчин, страдающих АГ: количество жировой ткани $-18,17$ кг $(\mathrm{U}=$ $367,0, Z=-3,05, p=0,002)$, мышечной ткани $-35,8$ кг $(U=100,0, Z=-6,06, p=$ $0,001)$, костной ткани $-13,35$ кг $(\mathrm{U}=282,5, \mathrm{Z}=-4,0, \mathrm{p}=0,001)$.

По показателям ОТ, ОТ/ОБ, ОТ/рост•100, ИВО, ИПНЛ, ТГ/ЛПВП среди обследуемых статистически значимых различий выявлено не было. Наиболее низкие показатели ОТ $(96,0)$, ОТ/ОБ $(0,97)$, ОТ/рост•100 $(54,95)$ и ИВО $(1,36)$ наблюдались в группе мужчин, имеющих ХОБЛ и АГ. ИПНЛ был выше у мужчин с АГ $(41,8)$, чем у мужчин с ХОБЛ $(34,29)$, но различие между группами было статистически не значимо.

Проведен анализ компонентного состава массы тела у обследованных мужчин в зависимости от степени ХОБЛ (табл. 3).

Таблицуа 2.

Уровни артериального давления и показатели компонентного состава массы тела у обследованных мужчин $\left(\mathrm{Me}\left[\mathrm{Q}_{25}-\mathrm{Q}_{75}\right]\right)$

\begin{tabular}{|c|c|c|c|c|}
\hline $\begin{array}{c}\text { Изучаемые } \\
\text { показатели }\end{array}$ & $\begin{array}{c}1 . \text { ХОБЛ (+), } \\
\mathrm{AГ} \mathrm{(-)}(\mathrm{n}=29)\end{array}$ & $\begin{array}{c}2 . \text { ХОБЛ+АГ } \\
(\mathrm{n}=63)\end{array}$ & $\begin{array}{c}3 . \mathrm{AГ}(+), \\
\text { ХОБЛ }(-) \\
(\mathrm{n}=44)\end{array}$ & \multirow{2}{*}{$p$} \\
\cline { 1 - 4 } & $\mathrm{Me}[\mathrm{Q} 25-\mathrm{Q} 75]$ & $\mathrm{Me}[\mathrm{Q} 25-\mathrm{Q} 75]$ & $\mathrm{Me}[\mathrm{Q} 25-\mathrm{Q} 75]$ & \\
\hline \multirow{2}{*}{ САД (мм рт.ст.) } & $\begin{array}{c}120,0 \\
{[120,0-120,0]}\end{array}$ & $\begin{array}{c}130,0 \\
{[130,0-140,0]}\end{array}$ & $\begin{array}{c}130,0 \\
{[120,0-140,0]}\end{array}$ & $\begin{array}{l}\mathrm{p}_{1,2}=\mathbf{0 , 0 0 1} \\
\mathrm{p}_{1,3}=\mathbf{0 , 0 0 1} \\
\mathrm{p}_{2,3}=0,114\end{array}$ \\
\cline { 1 - 3 } ДАД (мм рт.ст.) & $\begin{array}{c}80,0 \\
{[80,0-80,0]}\end{array}$ & $\begin{array}{c}80,0 \\
{[80,0-90,0]}\end{array}$ & $\begin{array}{c}80,0 \\
{[80,0-90,0]}\end{array}$ & $\begin{array}{c}\mathrm{p}_{1,2}=\mathbf{0 , 0 0 1} \\
\mathrm{p}_{1,3}=\mathbf{0 , 0 0 5} \\
\mathrm{p}_{2,3}=0,224\end{array}$ \\
\hline
\end{tabular}


Окончание табл. 2.

\begin{tabular}{|c|c|c|c|c|}
\hline Длина тела (см) & $\begin{array}{c}172,0 \\
{[168,0-176,0]}\end{array}$ & $\begin{array}{c}172,0 \\
{[168,0-176,0]}\end{array}$ & $\begin{array}{c}175,5 \\
{[171,5-180,0]}\end{array}$ & $\begin{array}{l}\mathrm{p}_{1,2}=0,759 \\
\mathrm{p}_{1,3}=0,1726 \\
\mathrm{p}_{2,3}=\mathbf{0 , 0 2 7}\end{array}$ \\
\hline Масса тела (кг) & $\begin{array}{c}70,0 \\
{[60,0-82,0]}\end{array}$ & $\begin{array}{c}76,0 \\
{[65,0-90,0]}\end{array}$ & $\begin{array}{c}82,5 \\
{[76,0-98,5]}\end{array}$ & $\begin{array}{l}\mathrm{p}_{1,2}=0,129 \\
\mathrm{p}_{1,3}=\mathbf{0 , 0 0 1} \\
\mathrm{p}_{2,3}=\mathbf{0 , 0 1 6}\end{array}$ \\
\hline ИМТ (кг/м²) & $\begin{array}{c}23,37 \\
{[20,37-25,18]}\end{array}$ & $\begin{array}{c}25,1 \\
{[21,71-30,86]}\end{array}$ & $\begin{array}{c}27,96 \\
{[25,14-30,56]}\end{array}$ & $\begin{array}{l}\mathrm{p}_{1,2}=0,076 \\
\mathrm{p}_{1,3}=\mathbf{0 , 0 0 1} \\
\mathrm{p}_{2,3}=0,064\end{array}$ \\
\hline Жировая ткань (кг) & $\begin{array}{c}10,56 \\
{[4,84-16,64]}\end{array}$ & $\begin{array}{c}13,16 \\
{[8,11-21,92]}\end{array}$ & $\begin{array}{c}18,17 \\
{[10,42-24,10]}\end{array}$ & $\begin{array}{l}\mathrm{p}_{1,2}=0,137 \\
\mathrm{p}_{1,3}=\mathbf{0 , 0 0 2} \\
\mathrm{p}_{2,3}=0,056\end{array}$ \\
\hline $\begin{array}{l}\text { Мышечная ткань } \\
\text { (кг) }\end{array}$ & $\begin{array}{c}27,05 \\
{[22,8-28,96]} \\
\end{array}$ & $\begin{array}{c}26,58 \\
{[23,29-31,66]}\end{array}$ & $\begin{array}{c}35,8 \\
{[33,3-40,66]} \\
\end{array}$ & $\begin{array}{l}\mathrm{p}_{1,2}=0,357 \\
\mathrm{p}_{1,3}=\mathbf{0 , 0 0 1} \\
\mathrm{p}_{2,3}=\mathbf{0 , 0 0 1}\end{array}$ \\
\hline Костная ткань (кг) & $\begin{array}{c}11,03 \\
{[10,3-12,07]}\end{array}$ & $\begin{array}{c}10,72 \\
{[9,09-12,65]}\end{array}$ & $\begin{array}{c}13,35 \\
{[12,07-15,14]}\end{array}$ & $\begin{array}{l}\mathrm{p}_{1,2}=0,559 \\
\mathrm{p}_{1,3}=\mathbf{0 , 0 0 1} \\
\mathrm{p}_{2,3}=\mathbf{0 , 0 0 1}\end{array}$ \\
\hline OT & $\begin{array}{c}97,0 \\
{[88,0-110,0]} \\
\end{array}$ & $\begin{array}{c}96,0 \\
{[21,08-56,0]}\end{array}$ & $\begin{array}{c}98,0 \\
{[88,5-108,5]}\end{array}$ & $\begin{array}{l}\mathrm{p}_{1,2}=0,231 \\
\mathrm{p}_{1,3}=0,866 \\
\mathrm{p}_{2,3}=0,134\end{array}$ \\
\hline ОТ/ОБ & $\begin{array}{c}0,98 \\
{[0,95-1,04]}\end{array}$ & $\begin{array}{c}0,97 \\
{[0,92-1,01]}\end{array}$ & $\begin{array}{c}0,98 \\
{[0,95-1,03]}\end{array}$ & $\begin{array}{l}\mathrm{p}_{1,2}=0,114 \\
\mathrm{p}_{1,3}=0,64 \\
\mathrm{p}_{2,3}=0,216\end{array}$ \\
\hline ОТ/рост 100 & $\begin{array}{c}57,47 \\
{[50,55-62,5]}\end{array}$ & $\begin{array}{c}54,95 \\
{[49,11-60,36]}\end{array}$ & $\begin{array}{c}56,26 \\
{[50,58-63,08]}\end{array}$ & $\begin{array}{l}\mathrm{p}_{1,2}=0,253 \\
\mathrm{p}_{1,3}=0,757 \\
\mathrm{p}_{2,3}=0,386\end{array}$ \\
\hline ИВО & $\begin{array}{c}1,62 \\
{[0,98-2,79]}\end{array}$ & $\begin{array}{c}1,36 \\
{[1,01-2,04]}\end{array}$ & $\begin{array}{c}1,42 \\
{[0,85-2,03]}\end{array}$ & $\begin{array}{l}\mathrm{p}_{1,2}=0,425 \\
\mathrm{p}_{1,3}=0,335 \\
\mathrm{p}_{2,3}=0,827\end{array}$ \\
\hline ИПНЛ & $\begin{array}{c}34,29 \\
{[22,08-87,68]}\end{array}$ & $\begin{array}{c}36,48 \\
{[21,08-56,0]}\end{array}$ & $\begin{array}{c}41,8 \\
{[22,12-64,04]}\end{array}$ & $\begin{array}{c}\mathrm{p}_{1,2}=0,491 \\
\mathrm{p}_{1,3}=0,888 \\
\mathrm{p}_{2,3}=0,36\end{array}$ \\
\hline ТГ/ЛПВП & $\begin{array}{c}1,01 \\
{[0,64-1,65]}\end{array}$ & $\begin{array}{c}0,96 \\
{[0,71-1,42]}\end{array}$ & $\begin{array}{c}0,96 \\
{[0,63-1,47]}\end{array}$ & $\begin{array}{l}\mathrm{p}_{1,2}=0,765 \\
\mathrm{p}_{1,3}=0,769 \\
\mathrm{p}_{2,3}=0,955\end{array}$ \\
\hline
\end{tabular}

По среднему росту среди обследуемых статистически значимых различий не выявлено: у обследованных с ХОБЛ ІІ он составил 175,0 [169,0-182,0] см, с ХОБЛ III - 172,0 [167,0-175,0] см и с ХОБЛ IV - 170,0 [168,0-176,0] см соответственно. Статистически значимое увеличение длины тела наблюдается между группой мужчин без ХОБЛ $(175,5$ см) и больными с ХОБЛ III $(\mathrm{U}=505,0, \mathrm{Z}=2,4, \mathrm{p}=0,014)$ и ХОБЛ IV $(\mathrm{U}=547,5, \mathrm{Z}=2,2, \mathrm{p}=0,028)$. 
Наблюдалась тенденция к уменьшению массы тела с увеличением степени ХОБЛ: масса тела была выше у лиц с ХОБЛ II, и составила 80,0 $[70,0-88,0]$ кг в сравнении с ХОБЛ III - 75,0 [61,0 - 90,0] кг и ХОБЛ IV $68,0[60,0-83,0]$ кг, причем статистически значимое различие было между группами с ХОБЛ II и ХОБЛ IV ( $U=270,5, Z=2,1, p=0,036)$. Группа пациентов без ХОБЛ так же отличается большими показателями массы тела $82,5[76,0-98,5]$ кг в сравнении с мужчинами с ХОБЛ III (U=500,0, $Z=2,5$, $\mathrm{p}=0,013)$ и ХОБЛ IV $(\mathrm{U}=417,5, \mathrm{Z}=3,5, \mathrm{p}=0,001)$. Также среди мужчин с ХОБЛ II отмечались наибольшие показатели ИМТ - 24,76 [22,34 - 30,86] кг $/ \mathrm{M}^{2}$ в сравнении с пациентами, имеющими ХОБЛ III 24,66 [21,63 - 32,37]

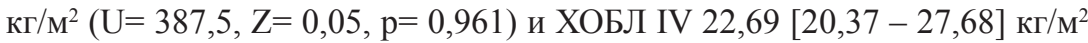
$(\mathrm{U}=300,0, \mathrm{Z}=1,6, \mathrm{p}=0,105)$ соответственно.

Таблища 3.

\section{Особенности компонентного состава массы тела у мужчин} в зависимости от степени тяжести ХОБЛ $\left(\mathrm{Me}\left[\mathrm{Q}_{25}-\mathrm{Q}_{75}\right]\right)$

\begin{tabular}{|c|c|c|c|c|c|c|}
\hline \multirow{2}{*}{$\begin{array}{l}\text { Изучае- } \\
\text { мые по- } \\
\text { казатели }\end{array}$} & $\begin{array}{c}\text { 1. ХОБЛ нет } \\
(\mathrm{n}=44)\end{array}$ & $\begin{array}{l}\text { 2. ХОБЛ II } \\
(\mathrm{n}=23)\end{array}$ & $\begin{array}{c}\text { 3. ХОБЛ III } \\
(\mathrm{n}=34)\end{array}$ & \begin{tabular}{|l} 
4. ХОБЛ ІV \\
$(\mathrm{n}=35)$
\end{tabular} & \multirow{2}{*}{\multicolumn{2}{|c|}{$\mathrm{p}$}} \\
\hline & $\mathrm{Me}$ [Q25-Q75] & $\mathrm{Me}$ [Q25-Q75] & $\mathrm{Me}[\mathrm{Q} 25-\mathrm{Q} 75]$ & $\mathrm{Me}[\mathrm{Q} 25-\mathrm{Q} 75]$ & & \\
\hline $\begin{array}{l}\text { Длина } \\
\text { тела, см }\end{array}$ & $\begin{array}{c}175,5 \\
{[171,5-180,0]}\end{array}$ & $\begin{array}{c}175,0 \\
{[169,0-182,0]}\end{array}$ & $\begin{array}{c}172,0 \\
{[167,0-175,0]}\end{array}$ & $\begin{array}{c}170,0 \\
{[168,0-176,0]}\end{array}$ & $\begin{array}{l}\mathrm{p}_{1,1}=0,953 \\
\mathrm{p}_{2,1,}=\mathbf{0 , 0 1 4} \\
\mathrm{p}_{2,3}=0,002\end{array}$ & $\begin{array}{l}\mathrm{p}_{1,4}=\mathbf{0 , 0 2 8} \\
\mathrm{p}_{2,4}=0,165 \\
\mathrm{p}_{3,4}=0,713\end{array}$ \\
\hline $\begin{array}{l}\text { Масса } \\
\text { тела, кг }\end{array}$ & $\begin{array}{c}82,5 \\
{[76,0-98,5]}\end{array}$ & $\begin{array}{c}80,0 \\
{[70,0-88,0]}\end{array}$ & $\begin{array}{c}75,0 \\
{[61,0-90,0]}\end{array}$ & $\begin{array}{c}68,0 \\
{[60,0-83,0]}\end{array}$ & $\begin{array}{l}\mathrm{p}_{1,2}=0,204 \\
\mathrm{p}_{1,3}=\mathbf{0 , 0 1 3} \\
\mathrm{p}_{2,3}=0,189\end{array}$ & $\begin{array}{l}\mathrm{p}_{1,4}=\mathbf{0 , 0 0 1} \\
\mathrm{p}_{2,4}=\mathbf{0 , 0 3 6} \\
\mathrm{p}_{3,4}=0,394\end{array}$ \\
\hline $\begin{array}{l}\text { ИМТ, } \\
\kappa г / \mathrm{M}^{2}\end{array}$ & $\begin{array}{c}27,96 \\
{[25,14-30,56]}\end{array}$ & $\begin{array}{c}24,76 \\
{[22,34-30,86]}\end{array}$ & $\begin{array}{c}24,66 \\
{[21,63-32,37]}\end{array}$ & {$\left[\begin{array}{c}22,69 \\
{[20,37-27,68]}\end{array}\right.$} & $\begin{array}{l}\mathrm{p}_{1,2}=0,097 \\
\mathrm{p}_{2,1,}=0,124 \\
\mathrm{p}_{2,3}=0,961\end{array}$ & $\begin{array}{l}\mathrm{p}_{1,4}=\mathbf{0 , 0 0 1} \\
\mathrm{p}_{2,4}=0,105 \\
\mathrm{p}_{3,4}=0,175\end{array}$ \\
\hline $\begin{array}{l}\text { Жировая } \\
\text { ткань, кг }\end{array}$ & $\begin{array}{c}18,17 \\
{[10,42-24,10]}\end{array}$ & $\begin{array}{c}13,46 \\
{[9,3-16,89]}\end{array}$ & $\begin{array}{c}12,38 \\
{[6,15-20,28]}\end{array}$ & $\begin{array}{c}10,56 \\
{[6,21-18,34]}\end{array}$ & $\begin{array}{l}\mathrm{p}_{1,2}=0,131 \\
\mathrm{p}_{1,3}=0,052 \\
\mathrm{p}_{2,3}=0,464\end{array}$ & $\begin{array}{l}\mathrm{p}_{1,4}=\mathbf{0 , 0 0 9} \\
\mathrm{p}_{2,4}=0,174 \\
\mathrm{p}_{3,4}=0,692\end{array}$ \\
\hline $\begin{array}{l}\text { Мы- } \\
\text { шечная } \\
\text { ткань, кг }\end{array}$ & $\begin{array}{c}35,79 \\
{[33,30-40,66]}\end{array}$ & $\mid \begin{array}{c}28,89 \\
{[26,33-31,31]}\end{array}$ & $\begin{array}{c}26,46 \\
{[22,8-29,24]}\end{array}$ & $\begin{array}{c}24,78 \\
{[21,74-29,85]}\end{array}$ & $\begin{array}{l}\mathrm{p}_{1,2}=\mathbf{0 , 0 0 1} \\
\mathrm{p}_{1,3}=\mathbf{0 , 0 0 1} \\
\mathrm{p}_{2,3}=0,067\end{array}$ & $\begin{array}{l}\mathrm{p}_{1,4}=\mathbf{0 , 0 0 1} \\
\mathrm{p}_{2,4}=\mathbf{0 , 0 1 2} \\
\mathrm{p}_{3,4}=0,294\end{array}$ \\
\hline $\begin{array}{l}\text { Костная } \\
\text { ткань, кг }\end{array}$ & $\begin{array}{c}13,35 \\
{[12,07-15,14]}\end{array}$ & $\begin{array}{c}12,27 \\
{[10,13-13,11]}\end{array}$ & $\begin{array}{c}10,99 \\
{[9,46-11,64]}\end{array}$ & $\begin{array}{c}10,43 \\
{[8,25-12,45]}\end{array}$ & $\begin{array}{l}\mathrm{p}_{1,1}=\mathbf{0 , 0 0 9} \\
\mathrm{p}_{1,3}=\mathbf{0 , 0 0 1} \\
\mathrm{p}_{2,3}=0,049\end{array}$ & $\begin{array}{l}\mathrm{p}_{1,4}=\mathbf{0 , 0 0 1} \\
\mathrm{p}_{2,4}=\mathbf{0 , 0 2} \\
\mathrm{p}_{3,4}=0,529\end{array}$ \\
\hline OT & $\begin{array}{c}98,0 \\
{[88,5-108,5]}\end{array}$ & $\begin{array}{c}96,0 \\
{[89,0-104]}\end{array}$ & $\begin{array}{c}97,0 \\
{[88,0-106,0]}\end{array}$ & $\begin{array}{c}92,0 \\
{[87,0-108,0]}\end{array}$ & $\begin{array}{l}\mathrm{p}_{1,2}=0,535 \\
\mathrm{p}_{1,3}=0,42 \\
\mathrm{p}_{2,3}=0,922\end{array}$ & $\begin{array}{l}\mathrm{p}_{1,4}=0,358 \\
\mathrm{p}_{2,4}=0,697 \\
\mathrm{p}_{3,4}=0,773\end{array}$ \\
\hline ОТ/ОБ & $\begin{array}{c}0,98 \\
{[0,95-1,03]}\end{array}$ & $\begin{array}{c}0,98 \\
{[0,92-1,02]}\end{array}$ & $\begin{array}{c}0,97 \\
{[0,94-1,04]}\end{array}$ & $\begin{array}{c}0,97 \\
{[0,93-1,01]}\end{array}$ & $\begin{array}{l}\mathrm{p}_{1,2}=0,501 \\
\mathrm{p}_{1,3}=0,732 \\
\mathrm{p}_{2,3}=0,580\end{array}$ & $\begin{array}{l}\mathrm{p}_{1,4}=0,505 \\
\mathrm{p}_{2,4}=0,975 \\
\mathrm{p}_{3,4}=0,843\end{array}$ \\
\hline
\end{tabular}


Окончание табл. 3.

\begin{tabular}{|c|c|c|c|c|c|}
\hline $\begin{array}{l}\text { OT/ } \\
\text { рост•100 }\end{array}$ & $\mid \begin{array}{c}56,26 \\
{[50,58-63,08]}\end{array}$ & $\begin{array}{c}56,47 \\
{[50,27-58,18]}\end{array}$ & $\begin{array}{c}56,56 \\
{[50,29-62,5]}\end{array}$ & {$\left[\begin{array}{c}54,12 \\
{[49,08-61,76]}\end{array}\right.$} & \begin{tabular}{|l|l|}
$\mathrm{p}_{1,2}=0,544$ & $\mathrm{p}_{1,4}=0,584$ \\
$\mathrm{p}_{1,3}=0,948$ & $\mathrm{p}_{2,4}=1,000$ \\
$\mathrm{p}_{2,3}=0,666$ & $\mathrm{p}_{3,4}=0,679$ \\
\end{tabular} \\
\hline ИВО & $\begin{array}{c}1,42 \\
{[0,85-2,03]}\end{array}$ & 1,61 & $\begin{array}{c}1,34 \\
{[0,87-2,04]}\end{array}$ & $\begin{array}{c}1,62 \\
{[1,18-2,20]}\end{array}$ & \begin{tabular}{l|l}
$\mathrm{p}_{1,2}=0,513$ & $\mathrm{p}_{1,4}=0,41$ \\
$\mathrm{p}_{1,3}=0,899$ & $\mathrm{p}_{2,4}=0,836$ \\
$\mathrm{p}_{2,3}=0,421$ & $\mathrm{p}_{3,4}=0,328$ \\
\end{tabular} \\
\hline ИПНЛ & $\left|\begin{array}{c}41,8 \\
{[22,12-64,04]}\end{array}\right|$ & $\begin{array}{c}41,18 \\
{[22,43-68,25]}\end{array}$ & $\begin{array}{c}35,73 \\
{[22,05-51,66]}\end{array}$ & $\begin{array}{r}36, \\
{[15,6-}\end{array}$ & $\begin{array}{lll}\mathrm{p}_{1,2}=0,911 & \mathrm{p}_{1,4}=0,733 \\
\mathrm{p}_{1,3}=0,378 & \mathrm{p}_{2,4}=0,949 \\
\mathrm{p}_{2,3}=0,553 & \mathrm{p}_{3,4}=0,723\end{array}$ \\
\hline $\begin{array}{l}\text { ТГ/ } \\
\text { ЛПВП }\end{array}$ & $\begin{array}{c}0,96 \\
{[0,63-1,47]}\end{array}$ & $\begin{array}{c}1,09 \\
{[0,59-2,11]}\end{array}$ & $\begin{array}{c}1,01 \\
{[0,64-1,36]}\end{array}$ & $\begin{array}{c}0,96 \\
{[0,78-1,42]}\end{array}$ & \begin{tabular}{|l|l}
$\mathrm{p}_{1,2}=0,639$ & $\mathrm{p}_{1,4}=0,675$ \\
$\mathrm{p}_{1,3}=0,668$ & $\mathrm{p}_{2,4}=0,924$ \\
$\mathrm{p}_{2,3}=0,407$ & $\mathrm{p}_{3,4}=0,464$ \\
\end{tabular} \\
\hline
\end{tabular}

Установлено, что наименьшие значения ИМТ у больных с ХОБЛ отмечались у лиц с IV степенью и были статистически значимо ниже в сравнении с лицами без ХОБЛ: 22,69 [20,37 - 27,68] кг/ $\mathrm{M}^{2}$ vs 27,96 [25,14 - 30,56] $\kappa г / \mathrm{M}^{2}(\mathrm{U}=425,0, \mathrm{Z}=3.4, \mathrm{p}=0,001)$. Таким образом, в ходе прогрессирования ХОБЛ уменьшаются значения ИМТ, с параллельным увеличением доли пациентов с нормальной массой тела (за счет уменьшения доли больных с избыточной массой тела и ожирением). Полученные результаты согласуются с данными Лемишевской С.С. (2012) [12, с. 42-48] согласно которым нормальный ИМТ был у 10\% пациентов ХОБЛ I, у 20\% с ХОБЛ ІІ и у $60 \%$ больных с ХОБЛ III [12, с. 42-48]. Болотовой Е.В. с соавт. (2018) показано, что наименьшие средние величины ИМТ встречались у больных тяжелой и крайне тяжелой ХОБЛ [1, с. 453-459].

Значения общего количества жировой, мышечной и костной ткани у больных ХОБЛ II превышали аналогичные показатели больных с ХОБЛ ІІІ и ХОБЛ IV, при этом по абсолютному количеству мышечной и костной ткани наблюдались статистические значимые различия между ХОБЛ ІІ и ХОБЛ IV: $28,89[26,33-31,31]$ кг vs $24,78[21,74-29,85]$ кг $(U=243,5, Z=2,5, p=$ $0,012)$ и $12,27[10,13-13,11]$ кг vs $10,43[8,25-12,45]$ кг $(\mathrm{U}=255,5, Z=2,3$, $\mathrm{p}=0,019)$ соответственно. В группе мужчин без ХОБЛ отмечались статистически значимо наибольшие показатели абсолютного количества мышечной и костной ткани в сравнении с ХОБЛ II, ХОБЛ III и ХОБЛ IV.

По показателям ОТ, ОТ/ОБ, ОТ/рост•100, ИВО, ИПНЛ, ТГ/ЛПВП среди обследуемых статистически значимых различий между группами выявлено не было.

\section{Выводы}

Таким образом, в ходе проведенного исследования были выделены закономерности распределения конституциональных типов у мужчин с 
ХОБЛ в условиях коморбидности, а также установлены особенности антропометрических показателей и компонентного состава массы тела в исследуемых группах. Особенностью распределения мужчин по конституциональным типам является преобладание лиц с грудным конституциональным типом среди мужчин с ХОБЛ и преобладание лиц с мускульным конституциональным типом среди мужчин с АГ и ХОБЛ и АГ. Получены данные о росто-весовых параметрах, компонентном составе тела у представителей с ХОБЛ в зависимости от степени тяжести заболевания. При прогрессировании ХОБЛ отмечается тенденция к уменьшению ИМТ и снижению жировой и мышечной компонент массы тела.

\section{Список литературы}

1. Болотова Е.В., Дудникова А.В., Являнская В.В. Особенности исследования состава тела у больных хронической обструктивной болезнью легких // Пульмонология. 2018. Т.28, №4. С. 453-459.

2. Бунак В.В. Методика антропометрических исследований. М.: Медгиз, 1931. $222 \mathrm{c}$.

3. Вахламов В.А., Тюрикова А.В. Обоснование использования новых методов исследования метаболического синдрома в диагностике и лечении пациентов с бронхообструктивными заболеваниями // Современные технологии в медицине. 2015. Т.7, № 4/ С. 127-134.

4. Владимирова Я.Б. Приоритетные направления изучения компонентного состава тела человека с учетом этнической принадлежности // Системный анализ и управление в биомедицинских системах. 2015. Т.14. №2. C. $330-333$.

5. Глобальная стратегия диагностики, лечения и профилактики хронической обструктивной болезни легких (пересмотр 2014 г.) / Пер. с англ. под ред. А.С. Белевского. М.: Российское респираторное общество, 2014. 92 с.

6. Деревянных Е.В., Яскевич Р.А. Антропометрические особенности и компонентный состав массы тела у медицинских работников старших возрастных групп // Клиническая геронтология. 2018. Т. 24. № 9-10. С. 19-21.

7. Деревянных Е.В., Яскевич Р.А. Распространенность избыточной массы тела и ожирения среди медицинских работников старших возрастных групп // Клиническая геронтология. 2018. Т. 24. № 9-10. С. 21-23.

8. Деревянных Е.В., Яскевич Р.А., Балашова Н.А. Антропометрические особенности и компонентный состав массы тела у мужчин медицинских работников с артериальной гипертонией // Международный журнал прикладных и фундаментальных исследований. 2016. № 6-2. С. 252-256. 
9. Деревянных Е.В., Яскевич Р.А., Балашова Н.А., Москаленко О.Л. Распространенность избыточной массы тела среди женщин медицинских работников крупной клинической больницы города Красноярска // В мире научных открытий. 2016. № 7 (79). С. 26-46.

10. Козлов Е.В., Деревянных Е.В., Балашова Н.А. и др. Хроническая обструктивная болезнь легких как социально-экономическое бремя взрослого населения (научный обзор) // В мире научных открытий. 2018. Т. 10. № 3. C. $180-199$.

11. Лемешевская С.С. Значимость изучения компонентного состава тела при хронической обструктивной болезни легких // Медицинский журнал. 2014. № 3 (49). С. 14-19.

12. Лемешевская С.С. Компонентный состав тела и распределение жировой ткани у мужчин с хронической обструктивной болезнью легких // Военная медицина. 2012. № 3 (24). С. 42-48.

13. Никитюк Д.Б., Николенко В.Н., Хайруллин Р.М. и др. Антропометрический метод и клиническая медицина // Журнал анатомии и гистопатологии. 2013. Т. 2, №4. C. 10-14.

14. Солонго Х., Наранцэцэг Ж., Наранцэцэг Б. и др. Исследование висцерального жира у больных хронической обструктивной болезнью легких // Сибирский медицинский журнал (Иркутск). 2013. Т. 119. № 4. С. 60-63.

15. Прокопьев А.Н., Пономарева К.А. Соматотипологические особенности компонентного состава тела человека // Вестник Шадринского государственного педагогического института. 2013. № 1 (17). С. 14-19.

16. Танченко О.А., Нарышкина С.В. Ожирение, метаболические нарушения и артериальная гипертония у больных хронической обструктивной болезнью легких: современные представления о коморбидности (обзор литературы) // Бюллетень физиологии и патологии дыхания. 2016. № 59. С. 109-118.

17. Фалетрова С.В., Коршунова Л.В., Бельских Э.С. Клинико-функциональные особенности хронической обструктивной болезни легких у пациентов с ожирением // Наука молодых - Eruditio Juvenium. 2018. №3. С. 439-447.

18. Чазова И.Е., Чучалин А.Г., Зыков К.А., Ратова Л.Г. Диагностика и лечение пациентов с артериальной гипертонией и хронической обструктивной болезнью легких (Рекомендации Российского медицинского общества по артериальной гипертонии и Российского респираторного общества // Системные гипертензии. 2013. №1. С. 5-34.

19. Чаплыгина Е.В., Аксенова О.А., Вартанова О.Т. и др. Современные представления о конституции человека и ее значение для медицины // Современные проблемы науки и образования. 2014. № 5. С. 468. 
20. Чтецов В.П., Лутовинова Н.Ю., Уткина М.И. Опыт объективной диагностики соматических типов на основе измерительных признаков у мужчин // Вопросы антропологии. 1978. Вып. 58. С. 3-22.

21. Чтецов В.П., Негашева М.А., Лапшина Н.Е. Изучение состава тела у взрослого населения: методические аспекты // Вестник Московского университета. Серия 23: Антропология. 2012. № 2. С. 43-52.

22. Яскевич Р.А., Москаленко О.Л. Антропометрические особенности и компонентный состав массы тела у мужчин мигрантов Крайнего Севера с артериальной гипертонией // В мире научных открытий. 2016. № 10 (82). C. $10-34$.

23. Amato M.C., Giordano C., Galia M. et al. Visceral Adiposity Index: a reliable indicator of visceral fat function associated with cardiometabolic risk // Diabetes Care. 2010. N.33, pp. 920-922.

24. Beijers R.J.H.C.G., van de Bool C., van den Borst B. et al. Normal weight but low muscle mass and abdominally obese: implications for the cardiometabolic risk profile in chronic obstructive pulmonary disease // Journal of the American Medical Directors Association. 2017. V.18. N.6. S. 533-538.

25. Kahn H.S. The «lipid accumulation product» performs better than the body mass index for recognizing cardiovascular risk: a population-based comparison // BMC Cardiovascular Disorders. 2005. N.5. P. 26.

26. Lee L.W., Lin C.M., Li H.C. et al. Body composition changes in male patients with chronic obstructive pulmonary disease: Aging or disease process? // PLoS One. 2017. Vol.10. N.12 (7). P. e0180928.

27. Makarevich A.E., Lemiasheuskaya S. Dynamics of body composition in male patients during chronic obstructive pulmonary disease (COPD) development // Pneumonol Alergol Pol. 2015. V.83. N.6, pp. 424-30.

28. Spelta F., Fratta Pasini A.M., Cazzoletti L., Ferrari M. Body weight and mortality in COPD: focus on the obesity paradox // Eat Weight Disord. 2018. V.23. N.1. P.15-22.

29. Yaskevich R.A., Moskalenko O.L. Anthropometric characteristics and component composition of body weight in male migrants of the far north with arterial hypertension // В мире научных открытий. 2017. Т.9. № 4. С. 47-63.

\section{References}

1. Bolotova E.V., Dudnikova A.V., YAvlyanskaya V.V. Osobennosti issledovaniya sostava tela u bol'nyh hronicheskoj obstruktivnoj bolezn'yu legkih [Features of the study of body composition in patients with chronic obstructive pulmonary disease]. Pul'monologiya [Pulmonology]. 2018. V.28. №4, pp. 453-459. 
2. Bunak V.V. Metodika antropometricheskih issledovanij [Methods of anthropometric research]. M.: Medgiz, 1931. 222 p.

3. Vahlamov V.A., Tyurikova A.V. Obosnovanie ispol'zovaniya novyh metodov issledovaniya metabolicheskogo sindroma $\mathrm{v}$ diagnostike i lechenii pacientov $\mathrm{s}$ bronhoobstruktivnymi zabolevaniyami [Justification of the use of new methods of studying the metabolic syndrome in the diagnosis and treatment of patients with broncho-obstructive diseases]. Sovremennye tekhnologii v medicine [Modern technologies in medicine]. 2015. V.7. № 4, pp. 127-134.

4. Vladimirova Ya.B. Prioritetnye napravleniya izucheniya komponentnogo sostava tela cheloveka s uchetom ehtnicheskoj prinadlezhnosti [Priority areas for studying the component composition of the human body, taking into account ethnicity]. Sistemnyj analiz i upravlenie v biomedicinskih sistemah [System analysis and management in biomedical systems]. 2015. V.14. №2, pp. 330-333.

5. Global'naya strategiya diagnostiki, lecheniya i profilaktiki hronicheskoj obstruktivnoj bolezni legkih (peresmotr 2014 g.) [Global strategy for the diagnosis, treatment and prevention of chronic obstructive pulmonary disease (revision 2014)] / Ed. A.S. Belevsky. M.: Russian Respiratory Society. 2014. 92 p.

6. Derevyannyh E.V., Yaskevich R.A. Antropometricheskie osobennosti i komponentnyj sostav massy tela u medicinskih rabotnikov starshih vozrastnyh grupp [Anthropometric features and component composition of body weight in medical workers of the older age groups]. Klinicheskaya gerontologiya [Clinical gerontology]. 2018. V. 24. № 9-10, pp. 19-21.

7. Derevyannyh E.V., Yaskevich R.A. Rasprostranennost' izbytochnoj massy tela i ozhireniya sredi medicinskih rabotnikov starshih vozrastnyh grupp [The prevalence of overweight and obesity among medical workers of the older age groups]. Klinicheskaya gerontologiya [Clinical gerontology]. 2018. V. 24. № 9-10, pp. 21-23.

8. Derevyannyh E.V., Yaskevich R.A., Balashova N.A. Antropometricheskie osobennosti i komponentnyj sostav massy tela u muzhchin medicinskih rabotnikov $\mathrm{s}$ arterial'noj gipertoniej [Anthropometric features and component composition of body weight in men of medical workers with arterial hypertension]. Mezhdunarodnyj zhurnal prikladnyh i fundamental'nyh issledovanij [International Journal of Applied and Fundamental Research]. 2016. № 6-2, pp. 252-256.

9. Derevyannyh E.V., Yaskevich R.A., Balashova N.A., Moskalenko O.L. Rasprostranennost' izbytochnoj massy tela sredi zhenshchin medicinskih rabotnikov krupnoj klinicheskoj bol'nicy goroda Krasnoyarska [The prevalence of overweight among women medical workers of a large clinical hospital in Krasnoyarsk]. V mire nauchnyh otkrytij [In the world of scientific discoveries]. 2016. № 7 (79), pp. 26-46. 
10. Kozlov E.V., Derevyannyh E.V., Balashova N.A. i dr. Hronicheskaya obstruktivnaya bolezn' legkih kak social'no-ehkonomicheskoe bremya vzroslogo naseleniya (nauchnyj obzor) [Chronic obstructive pulmonary disease as a socio-economic burden of the adult population (scientific review)]. V mire nauchnyh otkrytij [In the world of scientific discoveries]. 2018. V.10. № 3, pp. 180-199.

11. Lemeshevskaya S.S. Znachimost' izucheniya komponentnogo sostava tela pri hronicheskoj obstruktivnoj bolezni legkih [The significance of studying body composition in chronic obstructive pulmonary disease]. Medicinskij zhurnal [Medical Journal]. 2014. № 3(49), pp. 14-19.

12. Lemeshevskaya S.S. Komponentnyj sostav tela i raspredelenie zhirovoj tkani u muzhchin s hronicheskoj obstruktivnoj bolezn'yu legkih [Component body composition and distribution of adipose tissue in men with chronic obstructive pulmonary disease]. Voennaya medicina [Military Medicine]. 2012. № 3(24), pp. 42-48.

13. Nikityuk D.B., Nikolenko V.N., Hajrullin R.M. i dr. Antropometricheskij metod i klinicheskaya medicina [Anthropometric method and clinical medicine]. Zhurnal anatomii i gistopatologii [Journal of Anatomy and Histopathology]. 2013. V.2. №4, pp. 10-14.

14. Solongo H., Narancehcehg ZH., Narancehcehg B. i dr. Issledovanie visceral'nogo zhira u bol'nyh hronicheskoj obstruktivnoj bolezn'yu legkih [Study of visceral fat in patients with chronic obstructive pulmonary disease]. Sibirskij medicinskij zhurnal (Irkutsk) [Siberian Medical Journal (Irkutsk)]. 2013. V.119. № 4, pp. 60-63.

15. Prokop'ev A.N., Ponomareva K.A. Somatotipologicheskie osobennosti komponentnogo sostava tela cheloveka [Somatotypological features of the component composition of the human body]. Vestnik SHadrinskogo gosudarstvennogo pedagogicheskogo institute [Bulletin of the Shadrinsk State Pedagogical Institute]. 2013. №1 (17), pp. 14-19.

16. Tanchenko O.A., Naryshkina S.V. Ozhirenie, metabolicheskie narusheniya i arterial'naya gipertoniya u bol'nyh hronicheskoj obstruktivnoj bolezn'yu legkih: sovremennye predstavleniya o komorbidnosti (obzor literatury) [Obesity, metabolic disorders and arterial hypertension in patients with chronic obstructive pulmonary disease: modern ideas about comorbidity (literature review)]. Byulleten'fiziologii i patologii dyhaniya [Bulletin of physiology and pathology of respiration]. 2016. № 59, pp. 109-118.

17. Faletrova S.V., Korshunova L.V., Bel'skih Eh.S. Kliniko-funkcional'nye osobennosti hronicheskoj obstruktivnoj bolezni legkih u pacientov s ozhireniem [Clinical and functional features of chronic obstructive pulmonary disease in patients with obesity]. Nauka molodyh-Eruditio Juvenium [Science of the Young Eruditio Juvenium]. 2018. №3, pp. 439-447. 
18. Chazova I.E., Chuchalin A.G., Zykov K.A., Ratova L.G. Diagnostika i lechenie pacientov s arterial'noj gipertoniej i hronicheskoj obstruktivnoj bolezn'yu legkih (Rekomendacii Rossijskogo medicinskogo obshchestva po arterial'noj gipertonii i Rossijskogo respiratornogo obshchestva [Diagnostics and treatment of patients with arterial hypertension and chronic obstructive pulmonary disease (Recommendations of the Russian Medical Society on arterial hypertension and the Russian Respiratory Society]. Sistemnye gipertenzii [Systemic hypertension]. 2013. №1, pp. 5-34.

19. Chaplygina E.V., Aksenova O.A., Vartanova O.T. i dr. Sovremennye predstavleniya o konstitucii cheloveka i ee znachenie dlya mediciny [Modern ideas about the constitution of man and its importance for medicine]. Sovremennye problemy nauki i obrazovaniya [Modern problems of science and education]. 2014. № 5. P. 468.

20. Chtecov V.P., Lutovinova N.YU., Utkina M.I. Opyt ob"ektivnoj diagnostiki somaticheskih tipov na osnove izmeritel'nyh priznakov u muzhchin [The experience of an objective diagnosis of somatic types on the basis of measuring signs in men]. Voprosy antropologii [Questions of anthropology]. 1978. Issue 58, pp 3-22.

21. Chtecov V.P., Negasheva M.A., Lapshina N.E. Izuchenie sostava tela u vzroslogo naseleniya: metodicheskie aspekty [The study of body composition in adults: methodological aspects]. Vestnik Moskovskogo universiteta. Seriya 23: Antropologiya [Moscow University Bulletin. Series 23: Anthropology]. 2012. №2, pp. 43-52.

22. Yaskevich R.A., Moskalenko O.L. Antropometricheskie osobennosti i komponentnyj sostav massy tela u muzhchin migrantov Krajnego Severa s arterial'noj gipertoniej [Anthropometric features and component composition of body weight in men of migrants of the Far North with arterial hypertension]. V mire nauchnyh otkrytij [In the world of scientific discoveries]. 2016. № 10(82), pp. 10-34.

23. Amato M.C., Giordano C., Galia M. et al. Visceral Adiposity Index: a reliable indicator of visceral fat function associated with cardiometabolic risk. Diabetes Care. 2010. N.33, pp. 920-922.

24. Beijers R.J.H.C.G., van de Bool C., van den Borst B. et al. Normal weight but low muscle mass and abdominally obese: implications for the cardiometabolic risk profile in chronic obstructive pulmonary disease. Journal of the American Medical Directors Association. 2017. V.18. N.6. 533-538.

25. Kahn H.S. The «lipid accumulation product» performs better than the body mass index for recognizing cardiovascular risk: a population-based comparison. $B M C$ Cardiovascular Disorders. 2005. N.5. P. 26.

26. Lee L.W., Lin C.M., Li H.C. et al. Body composition changes in male patients with chronic obstructive pulmonary disease: Aging or disease process? PLoS One. 2017. Vol.10. N.12 (7). P. e0180928. 
27. Makarevich A.E., Lemiasheuskaya S. Dynamics of body composition in male patients during chronic obstructive pulmonary disease (COPD) development. Pneumonol Alergol Pol. 2015. V.83. N.6. P. 424-30.

28. Spelta F., Fratta Pasini A.M., Cazzoletti L., Ferrari M. Body weight and mortality in COPD: focus on the obesity paradox. Eat Weight Disord. 2018. V.23. N.1, pp. 15-22.

29. Yaskevich R.A., Moskalenko O.L. Anthropometric characteristics and component composition of body weight in male migrants of the far north with arterial hypertension. V mire nauchnyh otkrytij. 2017. V.9. № 4, pp. 47-63.

\section{ДАННЫЕ ОБ АВТОРАХ}

Козлов Евгений Вячеславович, доцент кафедры пропедевтики внутренних болезней и терапии, заведующий пульмонологическим отделением, кандидат медицинских наук

ГБОУ ВПО «КрасГМУ им. проф. Ф.В. Войно-Ясенеикого» МЗ РФ; КГБУЗ «Красноярская межрайонная клиническая больница скорой медицинской помощи имени Н.С. Карповича» ул. Партизана Железняка, 1а, г. Красноярск, 660022, Российская Федераиия; ул. Курчатова, 17, г. Красноярск, 660062, Российская Федераиия kev-pulmonolog@mail.ru

Яскевич Роман Анатольевич, доцент кафедры пропедевтики внутренних болезней и терапии, ведущий научный сотрудник группы патологии сердечно-сосудистой системы, кандидат медицинских наук, доцент ГБОУ ВПО «КрасГМУ им. проф. Ф.В. Войно-Ясенеикого» МЗ РФ; Федеральное государственное бюджетное научное учреждение «Научно-исследовательский институт медицинских проблем Севера» ул. Партизана Железняка, 1а, г. Красноярск, 660022, Российская Федерация; ул. Партизана Железняка, 32, г. Красноярск, 660022, Российская Федерация cardio@impn.ru

Москаленко Ольга Леонидовна, старший научный сотрудник, кандидат биологических наук Федеральное государственное бюджетное научное учреждение «Научно-исследовательский институт медииинских проблем Севера» ул. Партизана Железняка, 32, г. Красноярск, 660022, Российская Федерация gre-ll@mail.ru 
Кочергина Ксения Николаевна, студент

ГБОУ ВПО «КрасГМУ им. проф. Ф.В. Войно-Ясенеикого» МЗ РФ ул. Партизана Железняка, 1а, г. Красноярск, 660022, Российская Федерация

ko4ergina.ksen@gmail.com

\section{DATA ABOUT THE AUTHORS}

Kozlov Evgeny Vyacheslavovich, Associate Professor at Department of Propedeutics of Internal Diseases and Therapy, Head of Pulmonology Department, Candidate of Medical Science

Krasnoyarsk State Medical University named after Professor V.F. VoinoYasenetzkiy; Krasnoyarsk Interdistrict Clinical Hospital of Emergency Medical Care. N.S. Karpovich

1a, P. Zheleznyaka St., Krasnoyarsk, 660022, Russian Federation; 17, Kurchatov St., Krasnoyarsk, 660062, Russian Federation kev-pulmonolog@mail.ru

Yaskevich Roman Anatolyevich, Associate Professor at Department of Propedeutics of Internal Diseases and Therapy, Leading Researcher of the Group Pathology of the Cardiovascular System, Candidate of Medical Science, Docent

Krasnoyarsk State Medical University named after Professor V.F. Voino-Yasenetzkiy; Scientific Research Institute of medical problems of the North 1a, P. Zheleznyaka St., Krasnoyarsk, 660022, Russian Federation; 3g, P. Zheleznyaka St., Krasnoyarsk, 660022, Russian Federation cardio@impn.ru

Moskalenko Olga Leonidovna, Senior Researcher, Candidate of Biological Sciences

Scientific Research Institute of medical problems of the North 3g, P. Zheleznyaka St., Krasnoyarsk, 660022, Russian Federation gre-ll@mail.ru

Kochergina Ksenia Nikolaevna, student

Krasnoyarsk State Medical University named after Professor V.F. Voino-Yasenetzkiy

1a, P. Zheleznyaka St., Krasnoyarsk, 660022, Russian Federation ko4ergina.ksen@gmail.com 